\title{
Erratum
}

\section{C9ORF72 Hexanucleotide Repeat Number in Frontotemporal Lobar Degeneration: A Genotype-Phenotype Correlation Study}

Luisa Benussi, Giacomina Rossi, Michela Glionna, Elisa Tonoli, Elena Piccoli, Silvia Fostinelli, Anna Paterlini, Rosa Flocco, Diego Albani, Roberta Pantieri, Cristina Cereda, Gianluigi Forloni, Fabrizio Tagliavini, Giuliano Binetti and Roberta Ghidoni

[Journal of Alzheimer's Disease 38(4), 2014, 799-808, DOI 10.3233/JAD-131028]

On the first page, p. 799, the affiliation for author Roberta Pantieri were incorrect. The correct affiliation for Dr. Pantieri is:

Neurology Unit, IRCCS Institute of Neurological Sciences, Bellaria Hospital, Bologna, Italy 